Cette problématique, qui n'a rien d'une mode, interpelle le lecteur dans les fondements même de sa culture et va, cela est certain, susciter de nouvelles recherches.

Ce recueil, tel un long et sinueux fil d'Ariane, permet au lecteur moderne de retrouver le fil du sens usé, effiloché, peut-être même rompu en certains endroits, pour comprendre, au-delà des réaménagements de sens de ces notions prises une à une, le système de valeurs aussi mouvant que subtil dans lequel elles s'intègrent et s'entrecroisent.

\title{
BRENDA DUNN-LARDEAU, Université du Québec à Montréal
}

Nicola Panichi. Plutarchus Redivivus ? La Boétie e suoi interpreti seguito da Discorso di Stefano della Boétie Della schiavitù volontaria o il Contro Uno, tradotta nell' italiano idioma da Cesare Paribelli. Coll. « Dalla Rivoluzione francese al Risorgimento italiano », 2. Naples, Vivarium (Istituto italiano per gli studi filosofici), 1999. P. 130, vi, 79.

Nicola Panichi, bien connue des seiziémistes pour ses ouvrages sur Montchrétien ou sur Montaigne, a eu la judicieuse idée de célébrer le deuxième centenaire de la première traduction italienne du Contr'un, due à Cesare Paribelli, en proposant un fac-simile de cet opuscule de 85 pages, établi d'après le rarissime exemplaire du fonds Payen de la Bibliothèque nationale de France.

Voilà qui nous vaut un joli volume, très élégamment imprimé et présenté ${ }^{1}$, où cette version italienne, témoin tangible de la constante actualité — comme de la redoutable ductilité - du texte boétien, se voit précédée, ce qui ne gâche rien, d'une solide et savante étude, intitulée «Plutarchus redivivus ? La Boétie e i suoi interpreti », consacrée à la réception et à l'interprétation du Discours de la Servitude volontaire, depuis les origines (sa diffusion manuscrite sans — ou avec, selon certains critiques - l'assentiment de Montaigne), jusqu'aux éditions actuelles. Panorama au sein duquel trouve naturellement sa place (la première d'ailleurs, $p$. 11-18) la version établie par notre républicain napolitain en 1799. Paribelli, alors emprisonné pour raisons politiques dans une des «Bastilles» du royaume, a travaillé d'après le texte donné par une édition Coste tardive des Essais (1745 ou postérieure), dont il traduit également certaines notes (voir p. 99-103).

Il y a peu à ajouter à cette vaste enquête introductive, alimentée aux meilleures sources, bien informée et qui ajoute des éléments (les commentaires de G. A. Borgese, ou de Pancrazi) au fascicule La Boétie de la BEF. Toutes les difficultés provoquées par le refus de Montaigne d'assumer lui-même la publication du texte de son ami défunt, et dues à cette diffusion non désirée par le parti protestant, sont à la fois montrées par le menu et finement analysées. La fortune critique et politique du texte est ensuite fort bien retracée, de siècle en siècle, puis de génération révolutionnaire en génération révolutionnaire (p. 11-65). Un manuscrit du Discours, inconnu des derniers éditeurs (Desgraves, Bayard, et Gontarbert), signalé pourtant voilà dix ans par Jean-Paul Barbier ${ }^{2}$ dans les papiers du savoyard Jean 
Piochet, et acquis en 1998 par les A. D. de Chambéry (cote : série 1 J 279 [1]), a néanmoins été laissé de côté ; il présente des variantes nombreuses et intéressantes par rapport aux deux grandes versions connues jusqu'à présent (le ms. de Mesmes BnF.fr.839 et ses dérivés d'une part ; de l'autre, la version imprimée de Goulart, celle reproduite par Coste et traduite par Paribelli, ou donnée encore par le ms. fr. 20157 de la BnF. très proche - tout comme d'ailleurs la première édition séparée de 1577 , ce qu'il aurait fallu signaler — de cette lectio « protestante ») ; voilà qui aurait pu permettre de montrer que loin de Paris, au delà du cercle Corbinelli-Pinelli, on semble de part et d'autre des Alpes, s'intéresser de près au discours. Par ailleurs, N. Panichi n'a pas exploité notre étude des Cahiers Mennaisiens $\left(\mathrm{n}^{\circ} 25\right.$, 1991) qui lui aurait permis, comme elle l'a fort bien fait pour la traduction de Paribelli dans ses premières pages, de replacer dans son contexte historique et politique précis l'édition procurée par Lamennais en 1835 (les Lois de septembre limitant la liberté d'expression).

Après avoir montré la singulière fortune de ce Discours, étendard tour à tour des Huguenots menacés par les rois très catholiques, des citoyens français de 1789 (ou napolitains de 1799), des adversaires de Louis-Philippe, des exilés de 1852, des anarchistes fin de siècle ou des Résistants de la Seconde guerre mondiale, après en avoir souligné la variété d'interprétation, N. Panichi propose, sous l'invocation de Plutarque et de sa conception de l'amitié (d'où le titre retenu pour son étude), sa propre lecture du texte (p. 65-97). Et ce n'est pas le moment le moins stimulant de cette ample préface, puisqu'on nous y présente avec justesse et finesse un La Boétie à la recherche de la «nature intrinsèque » (p. 73-74) du pouvoir politique, dont il découvre les origines non plus au ciel, comme tant de ses contemporains, mais sur terre, dans les replis opaques de la psychologie des peuples, ou plus exactement de chaque sujet. Car la servitude n'est pas un vol — vol de la liberté perpétré dans la violence et la contrainte par le tyran et ses hommes de main -, mais un don, une scandaleuse aliénation de sa propre liberté entre les mains du despote, librement consentie par chacun.

Après ces analyses qui synthétisent les principales interprétations auxquelles le Discours donne actuellement lieu, vient enfin (p. 105-30) une très solide bibliographie classée par ordre chronologique de publication, très à jour et fort complète. Voilà donc un bel et bon volume ; paradoxalement, en proposant au public italien de redécouvrir la plus ancienne version du texte de La Boétie (à laquelle, plutôt que de se référer à l'édition de M. Smith, l'introduction aurait dû logiquement renvoyer ${ }^{3}$ ), il constitue l'édition de loin la meilleure et la mieux informée des sept traductions publiées en Italie depuis 1994 (voir p. 108), où le Discours suscite visiblement un intérêt bien plus fort qu'en France ; et il permettra également de réfléchir au singulier destin du Contr'un : au milieu des périodes de crise cette declamatio humaniste a toujours su trouver des lecteurs qui y ont, siècle après siècle, puisé un vigoureux remède pour tenter de conjurer les malheurs de l'oppression.

MICHEL MAGNIEN, Université Paris III - Sorbonne Nouvelle 
90 / Renaissance and Reformation / Renaissance et Réforme

\section{Notes}

1. Fort peu de coquilles en effet à signaler : p. 41, 1. 2, Corbin pour Sorbin ; p. 63, 1. 1, succintly pour succinctly ; p. 97, n. 224, Qamvis pour Quamvis ; p. 103, dernière 1., extremenent (mais Payen a en fait écrit excessi/vement : v. son annotation ms. sur le f. de garde, p. 132) ; p. 105, 1. 7, Dupuys ; p. 111, 1. 33, libre pour livre.

2. Ma Bibliothèque poétique, Genève, Droz, t. II, 1990, p. 326. Les 8 vol. mss. ont été préemptés à Drouot lors de la vente du 30 janv. 1998 (v. cat. Bibliothèque d'un amateur savoyard, $\mathrm{n}^{\mathrm{0}}$ 67, p. 23-25, avec reproduction de la I ̀̀re page du Discours).

3. Le fort cloisonnement entre l'introduction et le texte italien proposé ensuite est le seul reproche que l'on puisse adresser à cette publication ; comme si cette reproduction anastatique était le prétexte à une synthèse sur La Boétie, alors qu'elle aurait dû en être le pré-texte, au sens étymologique. On aurait ainsi aimé que Mme Panichi portât un jugement circonstancié (plus développé que les minces relevés des p. 102-103) sur les qualités de la traduction de Paribelli. Le républicain s'y montre-t-il, paix à ses cendres, traditore ? Les collations rapides auxquelles nous nous sommes livré, mais notre avis est infiniment moins autorisé que celui d'une italophone, indiquent que le texte de La Boétie est rendu avec vigueur et fidélité, beaucoup mieux, paradoxalement, que dans l'adaptation en français moderne publiée à Paris en 1789 (BEF. no 84).

James J. Supple. Les Essais de Montaigne. Méthode(s) et méthodologies. Études montaignistes, 36. Paris, H. Champion. P. 431.

À partir des mises en garde formulées par J.-Y. Pouilloux dans Lire les Essais de Montaigne (Maspéro, 1969), une nouvelle critique montaigniste s'est dressée contre la lecture autobiographique et moralisante qui s'appuyait sur un «florilège » de passages épars ordonnés à dessein par le critique. J. J. Supple publie alors Arms versus Letters. The Military and Literary Ideals in the "Essais » of Montaigne (Oxford, Clarendon Press, 1984), ouvrage sévèrement critiqué par cette «nouvelle vague » lors de sa parution. Dans le présent ouvrage, il leur répond (p. ex., p. 80 : « la critique traditionaliste [...] n'a nullement besoin de rester sur la défensive »). Il structure, donc, cette étude de façon à démontrer les lacunes - et apports éventuels - de chaque « chapelle » en vue d'une connaissance approfondie d'essais spécifiques (Première et Deuxième Parties), de regroupements d'essais et de l'œuvre entière (Troisième Partie). De fait, il singularise l'œuvre de chaque chercheur en soulignant ses spécificités.

La graphie «méthode(s)» du sous-titre de cet ouvrage indiquerait la volonté d'expliciter la (ou les) méthode(s) de dispositio et d'elocutio constituant les Essais. Les « méthodologies » sont celles des critiques (traditionnelles et modernes) que notre collègue se donne pour tâche d'analyser. Ainsi, au fil de ses chapitres et grâce à une compréhension plus approfondie des diverses lectures, on serait amené à « se mettre de plein-pied » (p. 99) avec l'œuvre pour mieux discerner la (les) méthode(s) dont Montaigne se serait servi pour transmettre ses idées (« des idées précises » 